\title{
Neues aus der Kommission Vergleichende und Internationale Erziehungswissenschaft
}

\section{Jahrestagung zum Thema "Jenseits des Nationalen?"}

Die Jahrestagung „Jenseits des Nationalen? Erziehungswissenschaftliche Perspektiven" nimmt das gegenwärtig besonders ausgeprägte Spannungsverhältnis zwischen der Entgrenzung des Nationalen und Tendenzen der Re-Nationalisierung zum Anlass für eine gemeinsame Auseinandersetzung. Mit der Entgrenzung des Nationalen ist auf der einen Seite die Beobachtung gemeint, dass sich Lebensweisen und Identitäten zunehmend transnational bzw. in einer Dezentrierung essentialistischer Ideen von Kultur, Nation und Ethnizität ereignen und inter-, supra- oder transnationale Institutionen Einfluss auf national verstandene Kontexte haben. ReNationalisierung rekurriert auf der anderen Seite auf das Erstarken von (antimuslimischem) Rassismus und Antisemitismus, die Zunahme rechtspopulistischer Bewegungen sowie den Erfolg rechter Parteien.

Die Tagungsgebühr beträgt: 50,00€ Beschäftigte, 25,00€ Teilzeitbeschäftigte, $10,00 €$ Studierende, 10,00€ Nichterwerbstätige.

Sabine Hornberg

doi.org/10.31244/zep.2018.04.09

\section{Paolo-Freire-Kongress 2018}

Unter dem Titel „Dialogisches Denken und Bildung als Praxis der Freiheit" veranstaltete die Paulo-Freire-Kooperation e.V. (PFK) zusammen mit der Fakultät für Erziehungswissenschaften der Universität Hamburg vom 9. bis 11. November 2018 einen Internationalen Kongress zur Freire-Pädagogik. Teilgenommen haben etwa 100 Praktiker/-innen aus der Erwachsenenbildung, der außerschulischen Jugendbildung, der Schule und der Sozialarbeit sowie Studierende und Lehrende an Hochschulen aus Brasilien, USA, Schweiz, Belgien, Ägypten, Finnland und Deutschland.

Im Rückblick: An der Universität Hamburg hatte bereits Gottfried Hausmann, Prof. für Vergleichende Erziehungswissenschaften den Studierenden 1971 erstmals die Ideen Paulo Freires vorgestellt, dessen „Pädagogik der Unterdrückten“ gerade in deutscher Sprache erschienen war. Spontan bildete sich damals eine Arbeitsgruppe, die 1973 ihre Ergebnisse veröffentlichte (Zs. betrifft: Erziehung, 7, Die Methode Paulo Freire). Weitere Arbeitskreise und Initiativen waren schließlich nach und nach entstanden: AG SPAK, PFG/ Paulo Freire Gesellschaft in München, PFK/ Paulo Freire Kooperation in Oldenburg, PFI/ Paulo Freire Institut in Berlin und der Paulo-Freire-Verlag in Oldenburg. Im November 2003 organisierte die PFK einen Kongress in Oldenburg „Das Menschenrecht auf Bildung für Alle“. Kürzlich im Oktober 2017 veranstaltete das PFI die Tagung in Berlin „Lernen im Dialog mit Lateinamerika - 20 Jahre in Bewegung mit Paulo Freire“.
Nun wieder in Hamburg wurde unter der Fragestellung, welche Anregungen Theorie und Praxis Paulo Freires zur Neuerfindung des eigenen Praxis- und Lernbereichs bieten, zum einen eine kritische Bestandsaufnahme des Wirkens von Freire in verschiedenen Ländern und Arbeitsbereichen vorgenommen. So war seine Arbeit als Stadtrat für Bildungsangelegenheiten von Sao Paulo (1989 bis 1993) ein gerade im deutschsprachigen Raum noch weitgehend unbekannter Schwerpunkt. Zum anderen beschäftigten sich die Teilnehmenden mit der Weiterentwicklung der Grundlagen einer Theorie der Dialogischen Pädagogik als Praxis der Freiheit und fragten dabei auch nach ergänzenden Theorien.

Ganz in diesem Sinne setzte sich zum Auftakt des Kongresses Prof. Dr. Christel Adick von der Ruhruniversität Bochum in ihrem Einführungsvortrag mit der Frage „Warum gerade Freire? Seine pädagogische und erziehungswissenschaftliche Bedeutung" auseinander: Ja, Freire gehört auch im deutschsprachigen Raum bereits zum Kanon, so ihr Befund. Im weiteren Verlauf des Kongresses gab es dann neben 8 offenen Diskussionsforen, in denen ein Drittel der Teilnehmenden im Rahmen von 15-minütigen „Inputs“ von ihren Arbeiten berichteten, auch drei Workshops zu praktischen Umsetzungen von Paulo Freires und auch Augusto Boals Ansätzen aus den Bereichen Theaterpädagogik und Kunsttherapie. Ganz im Sinne Freires dialektischen Verständnisses von Aktion und Reflexion ermöglichte dies schließlich eine Neubewertung der 